\title{
Targeted cancer therapy - present and future
}

\section{$\underline{\text { Levitzki A. }}$}

Hebrew University of Jerusalem, Jerusalem, Israel

Background: Targeted therapy is slowly replacing cytotoxic therapy of Cancer and is also implemented in non-malignant diseases. In Cancer the success of targeted therapy is mixed. In some cases like the utilization of Tamoxifen daily for 5 years, in women with breast cancer, the risk of dying from the disease drops by one third, compared to their chances without the drug. Woman who are Her2+ and treated with Herceptin are $36 \%$ less likely to have the cancer come back and are $40 \%$ less likely to die from cancer. Gleevec induces $89 \% 5$-year survival as compared to $30 \%$ in the pre-Gleevec era.
Improvement is expected with Dasatinib a dual Src/Bcr-Abl inhibitor. Gleevec reduces the 2-year recurrence of high risk GIST from $77 \%$ to $41 \%$. The performance of other targeted drug is less impressive but still benefits patients: Avastin, Tarceva, Sorafenib and Erbitux for example benefits patients for weeks to a few months and Plexxicon 4032 for a few months.

Thus, although targeted therapy has improved overall survival no long-term remission has been achieved except for cases in which the immune system has been harnessed in a targeted manner by using adoptive $\mathrm{T}$ cell therapy, which is very costly.

In the lecture I will discuss these issues and some novel strategies to upgrade targeted therapy. 